\title{
Paeoniflorin ameliorates rheumatoid arthritis in rat models through oxidative stress, inflammation and cyclooxygenase 2
}

\author{
ZHILIN JIA $^{1}$ and JIAO HE ${ }^{2}$ \\ Departments of ${ }^{1}$ Hematology and ${ }^{2}$ Library, The First Affiliated Hospital of Dalian Medical University, \\ Dalian, Liaoning 116011, P.R. China
}

Received January 7, 2015; Accepted October 9, 2015

DOI: $10.3892 /$ etm.2015.2908

\begin{abstract}
Paeoniflorin has anti-inflammatory, anti-allergy, immune regulatory and pain-relieving effects, amongst other roles. However, the mechanisms underlying the protective effects of paeoniflorin on rheumatoid arthritis (RA) remain under investigation; the objective of the current study was to evaluate these protective effects in the context of an RA model. Rats were randomly divided into 5 groups, as follows: The control group, the RA rat model group, and the paeoniflorin groups, in which paeoniflorin was administered at concentrations of 5, 10 and $20 \mathrm{mg} / \mathrm{kg}$ for 3 weeks. The pain thresholds and arthritic symptoms of the RA rats were measured. Oxidative stress and inflammatory cytokines were also analyzed and western blot analysis was used to evaluate cyclooxygenase-2 (COX-2) protein expression levels. Paeoniflorin significantly increased the pain threshold and decreased the arthritic symptoms in the RA rat model. Notably, paeoniflorin reduced the malondialdehyde concentration and increased the activity of superoxide dismutase, catalase and glutathione peroxidase. Furthermore, paeoniflorin attenuated the activity of nuclear factor- $\kappa \mathrm{B}$ p 65 unit, tumor necrosis factor- $\alpha$, interleukin (IL) $-1 \beta$ and IL- 6 , and reduced the COX-2 protein expression level. The present study indicates that paeoniflorin ameliorates disease in rat models of RA through oxidative stress, inflammation and alterations to COX-2 expression.
\end{abstract}

Correspondence to: Mr. Jiao He, Department of Library, The First Affiliated Hospital of Dalian Medical University, 222 Zhongshan Road, Dalian, Liaoning 116011, P.R. China

E-mail: jiaohe2014hj@163.com

Abbreviations: RA, rheumatoid arthritis; COX, cyclooxygenase; MDA, malondialdehyde; SOD, superoxide dismutase; CAT, catalase; GSH-Px, glutathione peroxidase; TNF- $\alpha$, tumor necrosis factor- $\alpha$; IL, interleukin

Key words: paeoniflorin, rheumatoid arthritis, oxidative stress, inflammatory, cyclooxygenase 2

\section{Introduction}

Rheumatoid arthritis (RA) is a systemic disease characterized by progressive synovitis and the degeneration of joints; however, the underlying pathogenesis of RA remains unclear (1). For patients with bone and joint damage caused by RA, the ultimate aim of treatment is to delay the disability of joint function caused by the disease (2). During active periods of RA, the hyperplastic synovial tissue and pannus directly erode articular cartilage and bone tissue surrounding the joints. Inhibiting the proliferation of inflammatory synovial tissue and inducing apoptosis in synovial tissue is therefore the primary aim of RA treatment (3).

Oxidative stress is closely associated with human aging, cardiovascular disease and chronic inflammation, amongst other diseases that have previously been associated with immune dysfunction. A complete antioxidant defense system is well-evolved, and includes antioxidant enzymes, antioxidants and a variety of other mechanisms tasked with damage repair and re-synthesis (4). The coordination and complementation of the various antioxidant defense systems in vivo guarantee their stable and effective involvement in the antioxidative stress effect. At present, the pathogenesis of RA remains to be elucidated, but it has previously been indicated that oxidative stress has an important role in the pathology of the disease (5).

RA is an inflammatory form of arthritis that may be caused by a variety of factors, including genetic or environmental causes and microbial invasion, amongst others. Tumor necrosis factor- $\alpha(\mathrm{TNF}-\alpha)$ and interleukin-1 $\beta$ (IL-1 $\beta$ ) are pro-inflammatory cytokines that are pivotal in the pathogenesis of RA (6). Cyclooxygenase (COX) is an enzyme necessary for the synthesis of prostaglandins, and a key rate-limiting enzyme in the initial steps of prostaglandin synthesis. In a previous study, COX-1 was suggested not to be directly involved in inflammation (7). However, another study has reported that COX-1 is not only involved in inflammation, but that it also aggravates inflammation, while COX-2 appears to be mainly involved in the early inflammatory processes, but has an anti-inflammatory effect during chronic inflammation (8).

Paeoniflorin is the main active constituent of peonies, used in traditional Chinese medicine, and is a monoterpene glycoside compound. Previous investigations of the pharmacological effects of paeoniflorin have revealed that paeoniflorin has multiple roles, which include the attenuation of free radical 
damage, the inhibition of intracellular calcium overload and the abrogation of neurotoxicity (9). In vivo experiments indicate that this has numerous biological effects, including a reduction in blood viscosity and platelet aggregation, dilation of blood vessels, improvement of microcirculation, inhibition of oxidation and action as an anti-convulsive, with low toxicity and few side effects (10). However, the mechanisms underlying the protective effects of paeoniflorin upon RA remain unclear. The present study therefore aimed to investigate the delayed protective effects of paeoniflorin in a rat model of RA, and to reveal the signaling pathways involved in the actions of paeoniflorin.

\section{Materials and methods}

Experimental rat model. Healthy, male, Sprague-Dawley rats weighing 250-300 g were obtained from the Animal Resource Center of the First Affiliated Hospital of Dalian Medical University (Dalian, China). The rats were maintained in individual cages under standard conditions (12:12-h light-dark cycle, $40-60 \%$ humidity and $22-24^{\circ} \mathrm{C}$ ), and provided with food and water ad libitum. All study protocols employed were in accordance with the guidelines of the Animal Care and Use Committee of the First Affiliated Hospital of Dalian Medical University.

Model establishment. The RA rat model was established as described previously (11). The experimental rats were placed in a cage with a fan in a high position (12:12-h light-dark cycle, $80-90 \%$ humidity, $4-8^{\circ} \mathrm{C}$ ) for 20 days. On the 21 st day of the experiment, rats were anesthetized with an intraperitoneal (i.p.) injection of $50 \mathrm{mg} / \mathrm{kg}$ sodium pentobarbital. Freund's complete adjuvant $(10 \mathrm{mg} / \mathrm{ml}$; F-5881; Sigma-Aldrich, St. Louis, MO, USA) was injected subcutaneously between the 2nd and 3rd toes of the right foot. The experimental rats were observed for 3 days and the right ankle demonstrated acute inflammatory swelling within $24 \mathrm{~h}$. Secondary, widespread arthritis occurred within $24 \mathrm{~h}$, manifesting in the forelimbs and contralateral limbs as red swellings or inflamed nodes; arthritis also spread to the ear and tail, indicating a successful model.

Grouping and treatment. The experimental rats were randomly divided into 5 groups. In the control $(\mathrm{Con} ; \mathrm{n}=8)$ and RA rat model (RA; $n=8)$ groups, the rats received sodium pentobarbital $(10 \mathrm{mg} / \mathrm{ml}$, i.p.), while in the paeoniflorin(5), (10) and (20) groups [Pae(5), Pae(10) and Pae(20), respectively; $\mathrm{n}=8$ in each], the rats were treated with 5,10 or $20 \mathrm{mg} / \mathrm{kg}$ paeoniflorin (i.p.), respectively, all for 3 weeks (12). The chemical structure of paeoniflorin (purity $>98 \%$; Nanjing University of Traditional Chinese Medicine, Institute of Chinese Material Medica, Nanjing, China) is indicated in Fig. 1.

Measurement of pain thresholds of the RA rat model. After a 3-week treatment with paeoniflorin, the pressure pain threshold $(\mathrm{g})$ was detected three times each session with an interval of 20 min between sessions, using an electronic pressure pain detector (Somedic AB, Hörby, Sweden), as previously described (13). The mean value was used to indicate the pressure pain threshold.

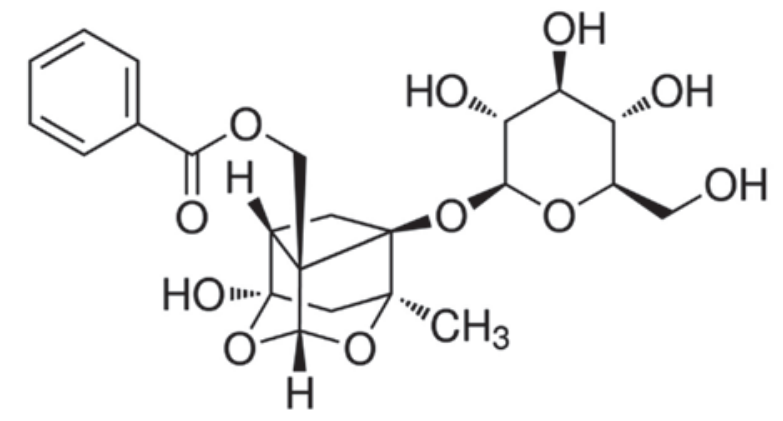

Figure 1. Chemical structure of paeoniflorin.

Clinical arthritic scoring of $R A$ rats. After the 3-week paeoniflorin treatment, the rats were evaluated for arthritis using a macroscopic scoring system, as follows: Severe arthritis of the entire paw and digits, 11-15 points; $>2$ joints involved, 6-10 points; 2 joints involved, $1-5$ points; and no signs of arthritis, 0 points.

Measurement of oxidative stress of $R A$ rats. After the 3-week paeoniflorin treatment, peripheral blood was collected. The blood samples were centrifuged at $3,000 \mathrm{x}$ g for $10 \mathrm{~min}$ at $4^{\circ} \mathrm{C}$, and were analyzed to detect the concentration of malondialdehyde (MDA) and the activity of superoxide dismutase (SOD), catalase (CAT) and glutathione peroxidase (GSH-Px), following the manufacturer's protocol (Beijing Boaosen Biotechnology, Ltd., Beijing, China).

Measurement of inflammatory effects on RA rats. Peripheral blood samples were processed as aforementioned, and the activity of nuclear factor (NF)- $\mathrm{kB}$ p65 unit, TNF- $\alpha, \mathrm{IL}-1 \beta$ and IL-6 were analyzed, following the manufacturer's protocol (Beijing Boaosen Biotechnology, Ltd.).

Western blot analysis of COX-2 in RA rats. Following treatment with paeoniflorin for 3 weeks, $10-\mathrm{mg}$ RA tissue samples were removed and incubated on ice for $30 \mathrm{~min}$ with $100 \mu \mathrm{l}$ tissue lysis buffer. Homogenates were centrifuged at $3,000 \mathrm{x} \mathrm{g}$ for $10 \mathrm{~min}$ at $4^{\circ} \mathrm{C}$ and protein concentration was measured using a bicinchoninic acid kit (Fermentas, Beijing, China). Equal protein was loaded onto $12 \%$ sodium dodecyl sulfate-polyacrylamide gels and transferred to polyvinylidene fluoride membranes (Millipore, Billerica, MA, USA). The following antibodies were used for the western blot analysis: Monoclonal anti-COX-2 (1:1,000; sc-376861) and anti- $\beta$-actin (1:500; sc-7210; Santa Cruz Biotechnology, Inc., Dallas, TX, USA) overnight at $4^{\circ} \mathrm{C}$. Membranes were incubated with anti-rabbit immunoglobulin $\mathrm{G}(\mathrm{IgG})$ horseradish peroxidase-conjugated secondary antibodies (Santa Cruz Biotechnology, Inc.) at $37^{\circ} \mathrm{C}$ for $1 \mathrm{~h}$. The relative band intensity was detected using the Amersham ECL Western Blotting Detection kit (GE Healthcare Buchler GmbH \& Co. KG, Braunschweig, Germany).

Statistical analysis. Data are expressed as the mean \pm standard deviation. Differences in arthritic score were evaluated by Student's t-test, and these were considered significant at $\mathrm{P}<0.05$. 


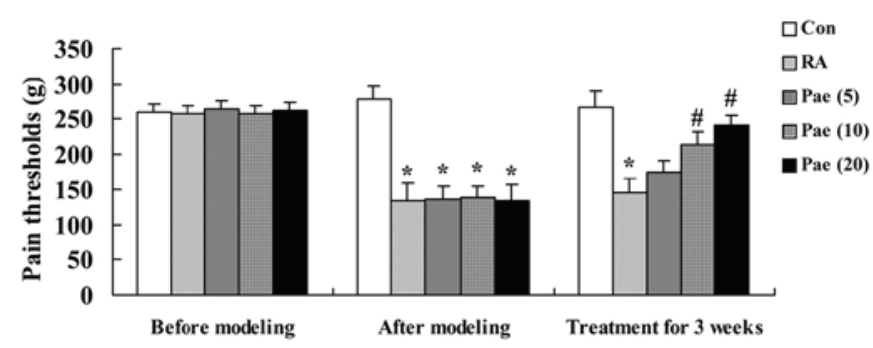

Figure 2. Effect of treatment with paeoniflorin on pain thresholds of the RA rat model. ${ }^{~} \mathrm{P}<0.01$ compared with the control group; ${ }^{\prime} \mathrm{P}<0.01$ compared with the RA group. Con, control; RA, rheumatoid arthritis; Pae, paeoniflorin; Pae(5), $5 \mathrm{mg} / \mathrm{kg}$-treated; Pae(10), $10 \mathrm{mg} / \mathrm{kg}$-treated; Pae(20), $20 \mathrm{mg} / \mathrm{kg}$-treated.

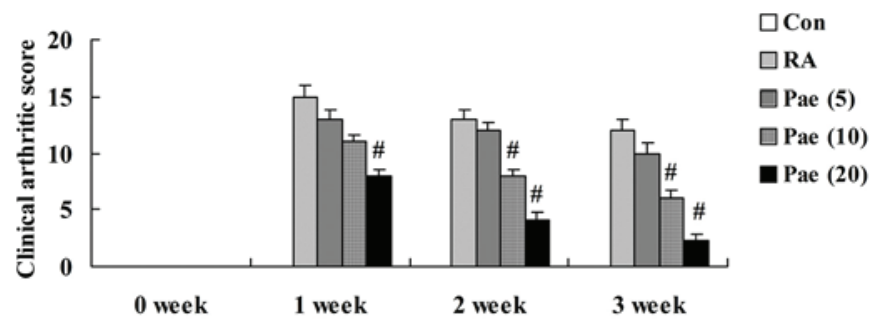

Figure 3. Effect of treatment with paeoniflorin on clinical arthritic score of the RA rat model. ${ }^{*} \mathrm{P}<0.01$ compared with the RA group. Con, control; RA, rheumatoid arthritis; Pae, paeoniflorin; Pae(5), $5 \mathrm{mg} / \mathrm{kg}$-treated; Pae(10), $10 \mathrm{mg} / \mathrm{kg}$-treated; Pae(20), $20 \mathrm{mg} / \mathrm{kg}$-treated.

\section{Results}

Effect of paeoniflorin on the pain thresholds of the RA rat model. The data in Fig. 2 demonstrates that 3 weeks of RA markedly reduced the pain threshold in the rats when compared with the control group. After the 3-week paeoniflorin treatment, 10 and $20 \mathrm{mg} / \mathrm{kg}$ of paeoniflorin were demonstrated to significantly recover the pain thresholds when compared with the $\mathrm{RA}$ rats $(\mathrm{P}<0.01$; Fig. 2$)$.

Effect of paeoniflorin on the clinical arthritic score of $R A$ rats. The clinical arthritic score of the $\mathrm{RA}$ model rats was markedly increased in comparison to the control group (Fig. 3). Treatment with $20 \mathrm{mg} / \mathrm{kg}$ of paeoniflorin significantly decreased the clinical arthritic score at 1,2 and 3 weeks of treatment when compared with that of the RA rats $(\mathrm{P}<0.01$; Fig. 3). Clinical arthritic score also significantly decreased following treatment with $10 \mathrm{mg} / \mathrm{kg}$ paeoniflorin for 2 and 3 weeks when compared with the RA rats $(\mathrm{P}<0.01$; Fig. 3$)$.

Effect of paeoniflorin on the concentration of MDA and the SOD, $C A T$ and GSH-Px activity in RA rats. To elucidate the antioxidant effects of paeoniflorin treatment in the RA model rats, the concentration of MDA, and the SOD, CAT and GSH-Px activity were measured. After the 3-week treatment period, the MDA concentration increased, and SOD, CAT and GSH-Px activity were reduced in the RA rats when compared with the control group (Fig. 4A-D). However, this effect was rescued following treatment with 10 and $20 \mathrm{mg} / \mathrm{kg}$ paeoniflorin (Fig. 4A-D).

Effect of paeoniflorin on $N F-\kappa B$ p 65 unit, TNF- $\alpha, I L-1 \beta$ and $I L-6$ activity in $R A$ rats. To elucidate the anti-inflammatory
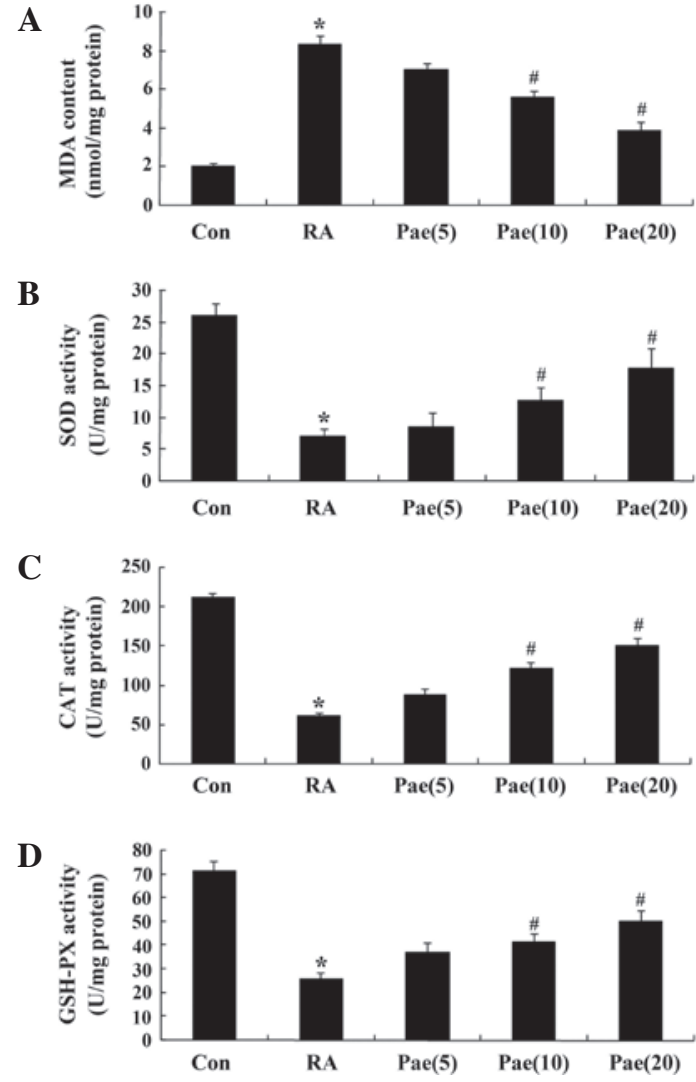

Figure 4. Effect of treatment with paeoniflorin on the concentration of (A) MDA, and activity of (B) SOD, (C) CAT and (D) GSH-Px in the RA rat model. ${ }^{*} \mathrm{P}<0.01$ compared with the control group; ${ }^{\prime \prime} \mathrm{P}<0.01$ compared with the RA group. Con, control; RA, rheumatoid arthritis; Pae, paeoniflorin; Pae(5), $5 \mathrm{mg} / \mathrm{kg}$-treated; Pae(10), $10 \mathrm{mg} / \mathrm{kg}$-treated; Pae(20), $20 \mathrm{mg} / \mathrm{kg}$-treated. SOD, superoxide mutase; CAT, catalase; GSH-Px, glutathione peroxidase; MDA, malondialdehyde.

effects of paeoniflorin treatment in RA rats, NF- $\kappa \mathrm{B}$ p65 unit, TNF- $\alpha$, IL-1 $\beta$ and IL- 6 activity were analyzed; activity was found to be significantly increased when compared with the control group (Fig. 5A-D). After a 3-week treatment with paeoniflorin (10 and $20 \mathrm{mg} / \mathrm{kg}$ ), NF- $\kappa \mathrm{B}$ p65 unit, TNF- $\alpha$, IL-1 $\beta$ and IL-6 activity was reduced in comparison with that of the RA rats $(\mathrm{P}<0.01$; Fig. 5A-D).

Effect of paeoniflorin on COX-2 in RA rats. As COX-2 has crucial roles in inflammation, the regulatory effects of paeoniflorin on the inflammatory response in the RA rats was examined. COX-2 protein expression was elevated in the RA rats compared with that of the control group (Fig. 6). Notably, paeoniflorin administration at 10 and $20 \mathrm{mg} / \mathrm{kg}$ significantly reduced COX-2 protein expression in the RA rat model $(\mathrm{P}<0.01$; Fig. 6$)$.

\section{Discussion}

RA is a symmetrical, chronic inflammatory disease primarily affecting multiple small peripheral joints, with possible extra-articular systemic damage (14). RA patients may suffer from pain, numbness, weight gain, difficulty in joint flexion and extension, joint swelling and a burning sensation in the muscles, bones and joints (15). In the present study, paeoniflorin 

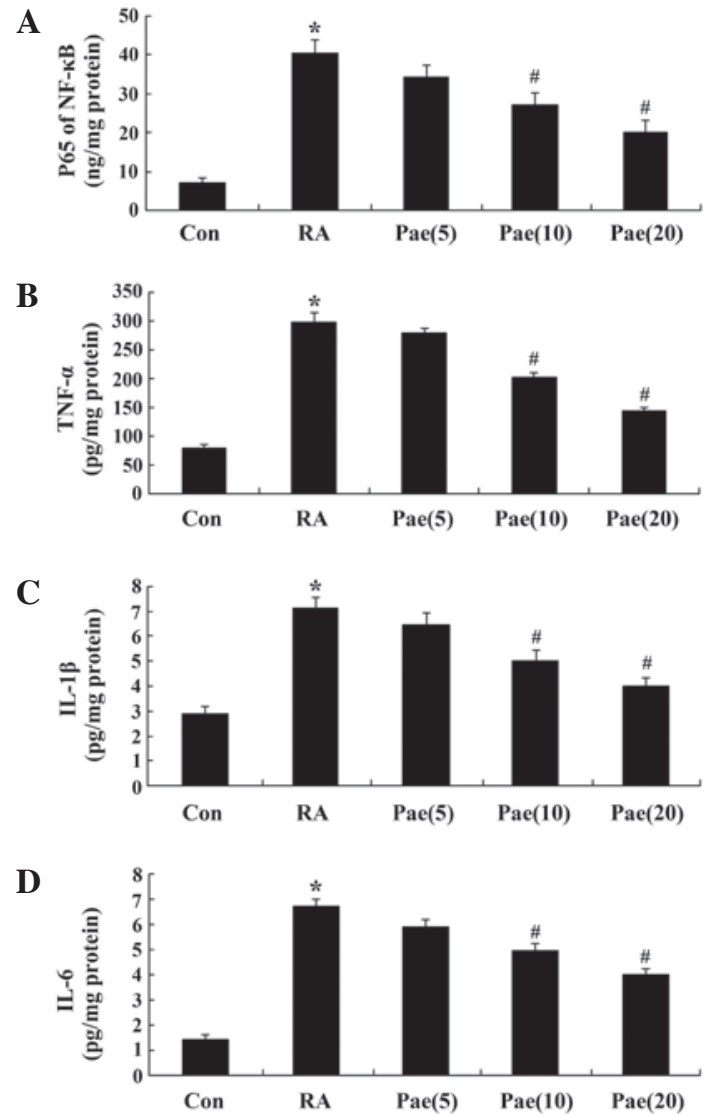

Figure 5. Effect of paeoniflorin treatment on the activity of (A) NF-кB p65 unit, (B) TNF- $\alpha,(C)$ IL-1 $\beta$ and (D) IL- 6 of the RA rat model. "P $<0.01$ compared with the control group; ${ }^{\#} \mathrm{P}<0.01$ compared with the RA group. Con, control; RA, rheumatoid arthritis; Pae, paeoniflorin; Pae(5), $5 \mathrm{mg} / \mathrm{kg}$-treated; Pae(10), $10 \mathrm{mg} / \mathrm{kg}$-treated; Pae(20), $20 \mathrm{mg} / \mathrm{kg}$-treated.; TNF- $\alpha$, tumor necrosis factor- $\alpha$; IL-1 $\beta$, interleukin-1 $\beta$; IL-6, interleukin-6.

significantly improved pain thresholds and reduced arthritic symptoms in an RA rat model. This is consistent with the results of a previous study by Zheng et al, which indicated that paeoniflorin suppressed arthritis in a rat model through its effects on synoviocytes, and by reducing COX-2 expression in the synovium (12). Paeoniflorin may therefore represent a potential therapeutic agent for the treatment of RA.

Within a normal mammalian body, the production and clearance of active oxygen are in a state of dynamic equilibrium. When the antioxidant system is dysfunctional, excessive accumulation of reactive oxygen species and associated metabolites occurs, causing tissue damage $(16,17)$. Previous studies have indicated that oxidative stress and RA are associated with elevated serum levels of lipid peroxidation reactant, a reduced level of SOD and the abnormal activity of antioxidant enzymes (18). In the present study, treatment with paeoniflorin decreased the MDA concentration and increased the SOD, CAT and GSH-Px activity in rats with RA. Similarly, Wankun et al demonstrated that paeoniflorin induces cellular apoptosis and protects ARPE-19 cells through repression of oxidative stress (19), and Zhao et al revealed that paeoniflorin protects against $\alpha$-naphthylisothiocyanate-induced cholestasis by ameliorating oxidative stress in rats (20).

Collagen-induced arthritis is primarily characterized by an early local inflammatory reaction, and secondary
A
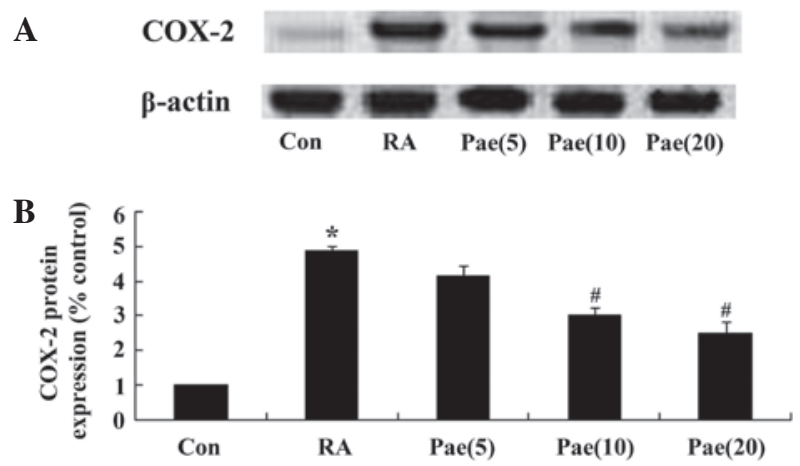

Figure 6. Effect of paeoniflorin treatment on (A) COX-2 protein expression levels using western blot analysis and (B) statistical analysis of COX-2 protein expression levels in the RA rat model. ${ }^{*} \mathrm{P}<0.01$ compared with the control group; $\mathrm{P}<0.01$ compared with the RA group. COX-2, cyclooxygenase-2; Con, control; RA, rheumatoid arthritis; Pae, paeoniflorin; Pae(5), $5 \mathrm{mg} / \mathrm{kg}$-treated; Pae(10), 10 mg/kg-treated; Pae(20), 20 mg/kg-treated.

lesions are manifested as contralateral hindlimb and forelimb swellings. When the synovial macrophages of RA patients are activated, the overexpression of inflammatory cytokines (including IL-1 $\beta$ and TNF- $\alpha$ ), chemokines (including IL-8 and macrophage inflammatory protein-1) and matrix metalloproteinases follows (21). The symptoms and degree of joint damage in RA are closely associated with the number of macrophages present, and with the levels of IL-1 $\beta$ and TNF- $\alpha$ (22). TNF- $\alpha$ induces endothelial cells to express adhesion molecules, and to promote leukocyte endothelial adhesion and tissue infiltration, resulting in local inflammation. In addition, TNF- $\alpha$ is able to promote cartilage cells to secrete plasminogen activator, transforming plasminogen into plasmin and thus accelerating arthritic damage. Furthermore, TNF- $\alpha$ can also induce synovial cells, macrophages, fibroblasts and chondrocytes to secrete IL-1 and IL-8, which increases tissue damage (23). The present study similarly demonstrated that paeoniflorin modulated the activity of NF- $\kappa$ B p 65 unit, TNF- $\alpha$, IL-1 $\beta$ and IL- 6 in the RA rat model. In previous associated studies, paeoniflorin induced anti-inflammatory effects in asthmatic mice (24) and inhibited the inflammatory response in mice presenting with allergic contact dermatitis (25).

COX-2 is an inducible chemical enzyme that is not typically expressed in numerous tissues (26). When the body is stimulated by proinflammatory cytokines, certain cells, including endothelial cells, vascular smooth muscle cells, monocytes macrophages and fibroblasts, are induced to express COX-2, such that COX-2 protein levels are rapidly upregulated between 8 - and 10-fold. COX-2 overexpression induces the synthesis and accumulation of prostaglandins, the inflammatory cytokines, in the damaged tissues, and promotes local inflammation and tissue damage (27). Overexpression of COX-2 can also promote cell proliferation, and inhibit apoptosis and the immune response, thereby evading immune surveillance, finally resulting in disruption of the balance between cell proliferation and apoptosis (28). In the current study, paeoniflorin significantly inhibited COX-2 protein expression. Similarly, a previous study also reported that paeoniflorin suppressed arthritis in a rat model by reducing COX-2 expression in the synovium (12), and another study 
revealed that paeoniflorin protected against ischemia-induced brain damage by inhibiting COX-2-mediated activity in rats (29).

In conclusion, the present findings demonstrate that the protective effect of paeoniflorin in RA treatment may occur through anti-oxidative and anti-inflammatory effects, and through the suppression of COX-2. Paeoniflorin may thus be considered a potential therapeutic agent in the treatment of RA, but more in-depth study is required to fully elucidate its mechanism and clinical effects.

\section{Acknowledgements}

The present study was supported by grants from the Research Foundation of Dalian Technology Bureau, China (grant no. 2012E15SF166) and Liaoning Province Science and Technology Plan Projects (grant no. 2013225002).

\section{References}

1. Dhaouadi T, Sfar I, Abelmoula L, Jendoubi-Ayed S, Aouadi H, Ben Abdellah T, Ayed K, Zouari R and Gorgi Y: Role of immune system, apoptosis and angiogenesis in pathogenesis of rheumatoid arthritis and joint destruction, a systematic review. Tunis Med 85: 991-998, 2007.

2. Wang K, Zhao L, Liu X, Hao Z, Zhou Y, Yang C and Li H: Differential co-expression analysis of rheumatoid arthritis with microarray data. Mol Med Rep 10: 2421-2426, 2014.

3. Liu H and Pope RM: The role of apoptosis in rheumatoid arthritis. Curr Opin Pharmacol 3: 317-322, 2003.

4. Radhakrishnan A, Tudawe D, Chakravarthi S, Chiew GS and Haleagrahara N: Effect of $\gamma$-tocotrienol in counteracting oxidative stress and joint damage in collagen-induced arthritis in rats. Exp Ther Med 7: 1408-1414, 2014.

5. Shahmohamadnejad S, Vaisi-Raygani A, Shakiba Y, Kiani A, Rahimi Z, Bahrehmand F, Shakiba E and Pourmotabbed T: Association between butyrylcholinesterase activity and phenotypes, paraoxonase192 rs662 gene polymorphism and their enzymatic activity with severity of rheumatoid arthritis: Correlation with systemic inflammatory markers and oxidative stress, preliminary report. Clin Biochem 48: 63-69, 2015.

6. Lu QY, Han QH, Li X, Li ZC, Pan YT, Liu L and Fu QG: Analysis of differentially expressed genes between rheumatoid arthritis and osteoarthritis based on the gene co-expression network. Mol Med Rep 10: 119-124, 2014.

7. Mederle K, Meurer M, Castrop H and Hocherl K: Inhibition of COX-1 attenuates the formation of thromboxane A2 and ameliorates the acute decrease in glomerular filtration rate in endotoxemic mice. Am J Physiol Renal Physiol 309: F332-F340, 2015.

8. Choi YJ, Lee WS, Lee EG, Sung MS and Yoo WH: Sulforaphane inhibits IL-1 $\beta$-induced proliferation of rheumatoid arthritis synovial fibroblasts and the production of MMPs, COX-2, and PGE2. Inflammation 37: 1496-1503, 2014.

9. Dong H, Li R, Yu C, Xu T, Zhang X and Dong M: Paeoniflorin inhibition of 6-hydroxydopamine-induced apoptosis in PC12 cells via suppressing reactive oxygen species-mediated $\mathrm{PKC} / \mathrm{NF}-\kappa \mathrm{B}$ pathway. Neuroscience 285: 70-80, 2015.

10. Choi EM, Suh KS, Rhee SY and Kim YS: Inhibitory effect of paeoniflorin on methylglyoxal-mediated oxidative stress in osteoblastic MC3T3-E1 cells. Phytomedicine 21: 1170-1177, 2014.
11. Luo L, Hu L, He L, Tang ZL, Song XG, Dirckinck-Holmfeld L and Cai RL: Effect of moxibustion on ultrastructure of synovial cells in rheumatoid arthritis rats. Zhen Ci Yan Jiu 36: 105-109, 2011 (In Chinese).

12. Zheng YQ, Wei W, Zhu L and Liu JX: Effects and mechanisms of Paeoniflorin, a bioactive glucoside from peony root, on adjuvant arthritis in rats. Inflamm Res 56: 182-188, 2007.

13. Zheng B, Hu L, Song X, Wu Z, Cai R, He L, Zhang C and Yu Q: Analgesic effect of different moxibustion durations in rheumatoid arthritis rats. J Tradit Chin Med 34: 90-95, 2014.

14. Zheng G, Wang L, Jia X, Li F, Yan Y, Yu Z, Li L, Wei Q and Zhang F: Application of high frequency color Doppler ultrasound in the monitoring of rheumatoid arthritis treatment. Exp Ther Med 8: 1807-1812, 2014.

15. Almoallim HM and Alharbi LA: Rheumatoid arthritis in Saudi Arabia. Saudi Med J 35: 1442-1454, 2014.

16. Baldeiras I, Santana I, Proença MT, Garrucho MH, Pascoal R, Rodrigues A, Duro D and Oliveira CR: Peripheral oxidative damage in mild cognitive impairment and mild Alzheimer's disease. J Alzheimers Dis 15: 117-128, 2008.

17. Hitchon CA and El-Gabalawy HS: Oxidation in rheumatoid arthritis. Arthritis Res Ther 6: 265-278, 2004.

18. Toyokuni S: Molecular mechanisms of oxidative stress-induced carcinogenesis: From epidemiology to oxygenomics. IUBMB Life 60: 441-447, 2008.

19. Wankun X, Wenzhen Y, Min Z, Weiyan Z, Huan C, Wei D, Lvzhen $\mathrm{H}, \mathrm{Xu} \mathrm{Y}$ and Xiaoxin L: Protective effect of paeoniflorin against oxidative stress in human retinal pigment epithelium in vitro. Mol Vis 17: 3512-3522, 2011.

20. Zhao Y, Zhou G, Wang J, Jia L, Zhang P, Li R, Shan L, Liu B, Song $\mathrm{X}$, Liu $\mathrm{S}$ and Xiao $\mathrm{X}$ : Paeoniflorin protects against ANIT-induced cholestasis by ameliorating oxidative stress in rats. Food Chem Toxicol 58: 242-248, 2013.

21. Klein K, Kabala PA and Grabiec AM: The bromodomain protein inhibitor I-BET151 suppresses expression of inflammatory genes and matrix degrading enzymes in rheumatoid arthritis synovial fibroblasts. Ann Rheum Dis: Dec 2, 2014 (Epub ahead of print).

22. Ichihara H, Yamasaki S, Hino M, Ueoka R and Matsumoto Y: Therapeutic effects of hybrid liposomes with downregulation of inflammatory cytokine for model mice of rheumatoid arthritis in vivo. Bioorg Med Chem Lett 25: 2686-2689, 2015.

23. Zhang J, Chen L, Delzell E, Muntner P, Hillegass WB, Safford MM, Millan IY, Crowson CS and Curtis JR: Republished: The association between inflammatory markers, serum lipids and the risk of cardiovascular events in patients with rheumatoid arthritis. Postgrad Med J 90: 722-729, 2014.

24. Sun J, Wu J, Xu C, Luo Q, Li B and Dong J: Paeoniflorin attenuates allergic inflammation in asthmatic mice. Int Immunopharmacol 24: 88-94, 2015.

25. Wang C, Yuan J, Wu HX, Chang Y, Wang QT, Wu YJ, Liu LH and Wei W: Paeoniflorin inhibits inflammatory responses in mice with allergic contact dermatitis by regulating the balance between inflammatory and anti-inflammatory cytokines. Inflamm Res 62: 1035-1044, 2013.

26. Yoon HY, Lee EG, Lee H, Cho IJ, Choi YJ, Sung MS, Yoo HG and Yoo WH: Kaempferol inhibits IL-1 $\beta$-induced proliferation of rheumatoid arthritis synovial fibroblasts and the production of COX-2, PGE2 and MMPs. Int J Mol Med 32: 971-977, 2013.

27. Dubois RN, Abramson SB, Crofford L, Gupta RA, Simon LS, Van De Putte LB and Lipsky PE: Cyclooxygenase in biology and disease. FASEB J 12: 1063-1073, 1998.

28. Markosyan N, Chen EP, Evans RA, Ndong V, Vonderheide RH and Smyth EM: Mammary carcinoma cell derived cyclooxygenase 2 suppresses tumor immune surveillance by enhancing intratumoral immune checkpoint activity. Breast Cancer Res 15: R75, 2013.

29. Guo RB, Wang GF, Zhao AP, Gu J, Sun XL and Hu G: Paeoniflorin protects against ischemia-induced brain damages in rats via inhibiting MAPKs/NF- $\kappa \mathrm{B}$-mediated inflammatory responses. PLoS One 7: e49701, 2012. 